\title{
Nonmagnetic and ferromagnetic fcc cerium studied with one-electron methods
}

\author{
Fabien Tran, Ferenc Karsai, and Peter Blaha \\ Institute of Materials Chemistry, Vienna University of Technology, \\ Getreidemarkt 9/165-TC, A-1060 Vienna, Austria
}

\begin{abstract}
Density functional theory was used to study the nonmagnetic (NM) and ferromagnetic (FM) phases of face-centered cubic cerium. Functionals of four levels of approximation for the exchangecorrelation energy were used: LDA, PBE, LDA/PBE $+U$, and YS-PBEh. The latter two contain an adjustable parameter, the onsite Coulomb repulsion parameter $U$ for $\mathrm{LDA} / \mathrm{PBE}+U$ and the fraction $\alpha_{\mathrm{x}}$ of Hartree-Fock exchange for YS-PBEh, which were varied in order to study their influence on the results. By supposing that, as a first approximation, the NM and FM solutions can be identified to the observed $\alpha$ and $\gamma$ phases, respectively, it is concluded that while a small value of $U$ or $\alpha_{\mathrm{x}}$ leads to the correct trend for the stability ordering of the two phases, larger values are necessary for a more appropriate (but still not satisfying) description of the electronic structure.

PACS numbers: 71.15.Ap, 71.15.Mb, 71.27.+a
\end{abstract}

\section{INTRODUCTION}

Cerium shows an isostructural (fcc $\rightarrow$ fcc) pressureinduced phase transition associated with a large $15 \%$ change of volume at room temperature $\stackrel{1}{\underline{1}}$ In the largevolume $\gamma$ phase, which is accessible above $\sim 200 \mathrm{~K}$, a Curie-Weiss behavior for the magnetic susceptibility is observed. Application of pressure drives cerium into the small-volume $\alpha$ phase, which shows Pauli paramagnetism. Many experimental and theoretical studies have been conducted in order to understand the mechanism underlying this phase transition. Essentially two models have been proposed. In the Mott transition model ${ }^{2}$ the $4 f$-electrons undergo a transition from a localized nonbonding character (in the $\gamma$ phase) to an itinerant bonding character (in the $\alpha$ phase), while the spd electrons are not considered to play any significant role. The other proposed mechanism is the Kondo volume collapse model ${ }^{3}$ in which hybridization between the $4 f$ and spd electrons is taken into account and leads to a screening of the local $4 f$ moment which is stronger in the $\alpha$ phase than in the $\gamma$ phase. Photoemission and bremsstrahlung isochromat spectroscopy experiments $\underline{4}^{\underline{-6}}$ showed that upper and lower Hubbard bands are present (i.e., the $4 f$ electrons are strongly correlated) in both phases, while a quasiparticle peak is observed only in the $\alpha$ phase, indicating a reduced strength of correlation in the $\alpha$ phase.

From a theoretical point of view, the approaches that have been used to study the $\alpha$ and/or the $\gamma$ phases include the local density (LDA) and generalized gradient (GGA) approximations, $\stackrel{7-12}{=}$ the self-interaction corrected LDA (SIC-LDA) method, $\stackrel{13-17}{=} \mathrm{LDA} / \mathrm{GGA}+U, \stackrel{18-21}{=} \mathrm{LDA}$

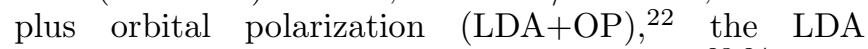
plus Gutzwiller approximation (LDA+GA) 23,24 LDA plus dynamic mean-field theory (LDA+DMFT), 21, 24-34 $G W \stackrel{35}{2}$ and a combined hybrid/Hartree-Fock+randomphase approximation (HF+RPA) study $\underline{12}$ (see Ref. 36 for a summary).

In general, the proper treatment of solids containing strongly correlated electrons with the Kohn-Sham (KS) equations ${ }^{37}$ of density functional theory (DFT) ${ }^{38}$ is not an easy task and, in particular, the results obtained with LDA or GGA are very often qualitatively incorrect. 39 Therefore, more advanced methods should be used for such solids, and most of them combine LDA (or GGA) with other theories (HF, DMFT, etc.). Since the $4 f$ electrons in the $\alpha$ phase of cerium are sometimes believed to be less localized (and therefore less strongly correlated) than in the $\gamma$ phase (in particular in the Mott picture mentioned above), then a fair description of the $\alpha$ phase could eventually be obtained with the semilocal (LDA/GGA) functionals. On the other hand, for a correct description of the more correlated $\gamma$ phase, a method beyond LDA/GGA, like DFT $+U$, has to be used.

In this work, we will present the results of a detailed DFT study on cerium. Four different levels of approximation for the exchange-correlation functional were used for the calculations: LDA, GGA, LDA/GGA $+U$, and GGA-hybrid. We will focus on the relative stability of the nonmagnetic (NM) and ferromagnetic (FM) phases of fcc cerium as well as on their electronic structures. As done in most of the previous DFT studies cited above, we will suppose that the NM and FM solutions of our calculations represent the experimentally observed $\alpha$ and $\gamma$ phases, respectively. However, it is not clear how legitimate such an identification can be considered. Actually, in the $\alpha$ phase it is not known exactly to what extent the local magnetic moment is quenched (the results of recent experiments suggest that the instantaneous moment remains stable across the transition ${ }^{40}$ ) and a more appropriate modelization of the paramagnetic $\gamma$ phase with static mean-field methods (like those used in the present work) should be done with a supercell containing randomly oriented moments, while all results of the present work were obtained in the one-atom unit cell. Obviously, the state-of-the-art method for such paramagnetic systems is LDA+DMFT, which treats more rigorously correlation effects. Therefore, this aspect of the calculations should be kept in mind when comparing our results with experiment.

In Refs. 32 and 41 it was concluded that at lowtemperature the $\alpha$ phase should be more stable than the 
$\gamma$ phase by $20-30 \mathrm{meV}$, and in Ref. 12 it was shown that the HF+RPA method is able to predict the correct stability ordering, while the hybrid functionals HSE06 ${ }^{42.43}$ and $\mathrm{PBE} 0 \stackrel{44.45}{ }$ can not. Actually, the correct stability ordering was previously reproduced with the LDA+OP (Ref. 22) and LDA+SIC (Ref. 17) methods, and in Ref. 20 it was shown that the GGA $+U$ method with a small value of $U$ also leads to the correct trend. At non-zero temperature, the LDA+DMFT method produces a depression in the total-energy curve, which is consistent with the volume collapse transition (see, e.g., Ref. 26). Here, we will show that the LDA/GGA $+U$ and GGA-hybrid methods lead to very similar results for the relative energy of the NM and FM phases as well as the electronic structure. In particular, by tunning the onsite Coulomb parameter or the fraction of HF exchange, the correct stability ordering can be obtained. However, none of the one-electron methods considered in this work (and in previous works), including the GGA-hybrid, is able to reproduce all features observed in the experimental spectra and therefore, as already concluded from previous works, it seems that the many-body effects seen in the spectra are such that they can not be mimicked by a one-electron method.

The present work is an attempt to give an overview of the (un)suitability of one-electron methods in general (KS and mixed KS/HF) to reproduce the experimental facts (lattice constant, stability ordering, and electronic properties) at low temperature.

The paper is organized as follows. In Sec. II, the theoretical method is briefly outlined and the computational details are given. In Sec. III the results are presented and discussed, and in Sec. IV] the summary is given.

\section{COMPUTATIONAL DETAILS}

The calculations were done with the all-electron WIEN2k code, $\stackrel{46}{ }$ which is based on the full-potential (linearized) augmented plane-wave plus local orbitals method 47 to solve the KS equations $\frac{37}{3}$ The one-atom fcc unit cell was used for the calculations and the integrations into the Brillouin zone were done with a $20 \times 20 \times 20$ k-mesh for the LDA, PBE, and LDA/PBE $+U$ functionals and a $12 \times 12 \times 12$ k-mesh for the much more expensive hybrid functional YS-PBEh. $\stackrel{48}{=} R_{\mathrm{MT}} K_{\max }=9(8$ for YS-PBEh), the product of the atomic sphere radius $R_{\mathrm{MT}}^{\mathrm{Ce}}=2.2 \mathrm{bohr}$ and the plane wave cutoff parameter $K_{\max }$, was used for the expansion of the basis set. An estimation of the error bar in our calculations for the relative energies of the $\mathrm{NM}$ and $\mathrm{FM}$ phases is $25 \mathrm{meV}$ for the hybrid functional YS-PBEh and $10 \mathrm{meV}$ for the other functionals. The symmetry constraint was reduced from cubic to orthorhombic, which is enough to lift the degeneracies of the $f$-orbitals and to allow an orbital moment to develop if spin-orbit coupling (SOC) is included. A symmetry breaking in Ce could also be explained by the fact that since Ce is paramagnetic, a Ce atom is surrounded by atoms with randomly oriented magnetic moments, which breaks the cubic symmetry. As a side remark, we note that if SOC is not included, then the electronic states NM and FM1 (see Sec. IIIA) can be reached with cubic symmetry, while FM2 and FM3 (for which also SOC is necessary) can not. All the presented results, including those on the NM phase, were obtained without imposing any constraint on the spin polarization.

Several functionals, namely LDA, PBE, $\mathrm{LDA}+U$, $\mathrm{PBE}+U$, and YS-PBEh, were considered for the present work. LDA is the exact functional for the uniform electron gas $\frac{37}{}$ and for the correlation part the analytical form of Perdew and Wang, ${ }^{49}$ PW92, was used. The PBE functional of Perdew, Burke, and Ernzerhof 50 is of the GGA form and is the most used functional for solid-state calculations. The LDA/PBE $+U$ functionals read

$$
E_{\mathrm{xc}}^{\mathrm{LDA} / \mathrm{PBE}+U}=E_{\mathrm{xc}}^{\mathrm{LDA} / \mathrm{PBE}}+E_{\mathrm{ee}}-E_{\mathrm{dc}},
$$

where $E_{\text {ee }}$ is an (rotationally invariant) electron-electron (ee) interaction energy of HF-type ${ }^{51.52}$ for the electrons of a selected atom and angular momentum $\ell(\ell=3$ for the $4 f$ electrons of cerium) and $E_{\mathrm{dc}}$ is the double-counting (dc) term for which we chose the fully localized limit

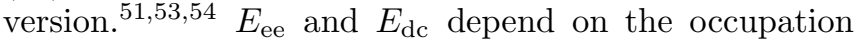
matrix $n_{m m^{\prime}}$. Since in cerium the number of $4 f$ electrons is one, the results depend mainly only on the difference $U-J$ between the Coulomb parameter $U$ and the exchange parameter $J$. Therefore, for simplicity we chose to set $J=0$ in all our calculations.

In the screened hybrid functional YS-PBEh, $\underline{48}$ (YS stands for Yukawa screened), a fraction $\alpha_{\mathrm{x}}(\in[0,1])$ of short-range (SR) PBE exchange is replaced by SR-HF exchange [usually, the acronym (YS)-PBE0 is used when $\left.\alpha_{\mathrm{x}}=0.25\right]$ :

$$
E_{\mathrm{xc}}^{\mathrm{YS}-\mathrm{PBEh}}=E_{\mathrm{xc}}^{\mathrm{PBE}}+\alpha_{\mathrm{x}}\left(E_{\mathrm{x}}^{\mathrm{SR}-\mathrm{HF}}-E_{\mathrm{x}}^{\mathrm{SR}-\mathrm{PBE}}\right),
$$

where $E_{\mathrm{x}}^{\mathrm{SR}-\mathrm{HF}}$ and $E_{\mathrm{x}}^{\mathrm{SR}-\mathrm{PBE}}$ are obtained from their unscreened counterparts by replacing the bare Coulomb operator by the exponentially attenuated Yukawa operator as proposed by Bylander and Kleinman .55 Note that the YS-PBEh functional leads to results which are very similar to the screened hybrid HSE06 ${ }^{42,43}$ functional which uses the complementary error function for the screening (see Ref. 48 for more details about YS-PBEh and Refs. 56 59 for recent applications). Spin-orbit coupling was included in the calculations using LDA, PBE, and $\mathrm{LDA} / \mathrm{PBE}+U$, but not YS-PBEh since at the moment it is not possible to include SOC in a calculation which uses the HF method. However, we could see that SOC has a small effect on the equilibrium lattice constants $(<0.01 \AA)$, relative energies $(<10 \mathrm{meV})$, and electronic structure. 


\section{RESULTS AND DISCUSSION}

\section{A. Occupation matrix}

Before starting the discussion of the results, we would like to point out the problem of the multiple solutions that can possibly be obtained with approximate functionals ${ }^{60}$ With functionals which lead to an orbitaldependent potential (e.g., DFT $+U$ or hybrid), multiple solutions can easily be obtained for systems with open $d$ or $f$-shell, and actually the $d$ - or $f$-orbitals occupation that is obtained at the end of the self-consistent field (SCF) procedure strongly depends on (and will eventually be more or less the same as) the orbitals occupation that is used to start the SCF procedure. 18,19 Therefore, with such functionals it is recommended to start a SCF calculation with each of the most plausible orbitals occupations in order to check which one leads to the lowest energy. In the following, we discuss the different states obtained with the various functionals.

Cerium has (about) one $4 f$ electron, and for the FM phase, among the solutions that we could stabilize with $\mathrm{PBE}+U$ (about ten, but probably more can be stabilized), the two lowest solutions consist of linear combinations of $Y_{3}^{-2}$ and $Y_{3}^{2}$, which is in accordance with Refs. 12, 17, and 19. The occupation matrices of these two FM solutions, called FM1 and FM2, are given in the Appendix $\mathrm{A}$ for the case $U=4.3 \mathrm{eV}$ including SOC. Note that FM1 and FM2 correspond (approximately since $\mathrm{SOC}$ is included) to an electron in the orbitals $f_{x y z}=$ $\left(Y_{3}^{2}-Y_{3}^{-2}\right) /(i \sqrt{2})$ and $f_{z\left(x^{2}-y^{2}\right)}=\left(Y_{3}^{2}+Y_{3}^{-2}\right) / \sqrt{2}$, respectively. It is noteworthy to mention that FM1 is the solution that was obtained by starting the $\mathrm{PBE}+U$ calculations from the PBE electron density, however, while FM1 is more stable than FM2 for small values of $U$, it is FM2 which is the most stable for larger values of $U$ (see details below). In Ref. 18, the solution corresponding to an electron mainly in $Y_{3}^{-2}$, reacheable only if SOC is included, was found to be the most stable with $\mathrm{LDA}+U$ among the states considered by the authors. It was possible to stabilize a similar state (called FM3) with $\mathrm{PBE}+U$, however as shown in Sec. IIIB it is less stable than FM1 and FM2. For the small-volume NM phase, only one solution could be stabilized and it corresponds to more or less equal occupancies of the diagonal terms as well as some off-diagonal terms [see Eq. (A4)]. The occupation matrix of the NM phase is rather similar among all functionals that we considered (from LDA to YS-PBEh). The spin-majority $4 f$-electron density is shown in Fig. 1. where we can see that it consists of 14 small lobes in the NM case [Fig. 11(d)], while there are 8 lobes for FM1 $\left[f_{x y z}\right.$, Fig. 1(a) $]$ and FM2 $\left[f_{z\left(x^{2}-y^{2}\right)}\right.$, Fig. 1(b)].

The FM1 and FM2 solutions could apparently not be stabilized with $\mathrm{LDA}+U$ when SOC is included, therefore, only NM and FM3 (the solution found in Ref. 18 with $\mathrm{LDA}+U)$ will be considered for this functional. However, we mention that the FM3 solutions obtained with $\mathrm{LDA}+U$ and $\mathrm{PBE}+U$ differ slightly in the sense that

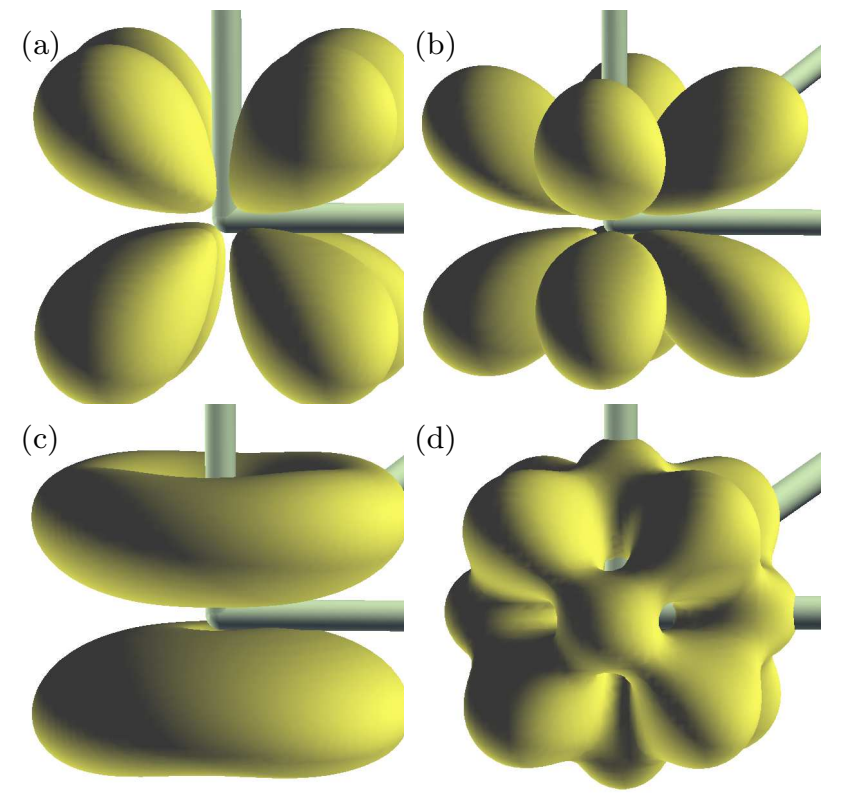

FIG. 1. (Color online) The spin-majority $4 f$-electron density at an isovalue of 0.1 electron/bohr ${ }^{3}$ for the FM1 (a), FM2 (b), FM3 (c), and NM (d) phases obtained from $\mathrm{PBE}+U$ with $U=4.3 \mathrm{eV}$ and SOC. The shown axis are those of the conventional cubic fcc unit cell.

with $\mathrm{PBE}+U$, all elements except $(-2,-2)$ of the occupation matrix are zero [see Eq. (A3)], while in the case of $\mathrm{LDA}+U$, the off-diagonal terms $( \pm 2, \mp 2)$ have a value of about 0.2. Therefore, the character of the FM3 solution obtained with $\mathrm{LDA}+U$ is intermediate between the FM2 and FM3 solutions obtained with $\mathrm{PBE}+U$. In a recent study 61 it has been shown that the gradient correction in GGA is responsible of the quenching of the orbital magnetic moment in $\mathrm{FeO}$ and $\mathrm{CoO}$. The same mechanism could eventually explain why the LDA $+U$ most stable FM solution (FM3) corresponds to a large orbital moment, while it is not the case with $\mathrm{PBE}+U$ since the orbital moments are much smaller in the case of FM1 and FM2 (results discussed in Sec. IIIC).

The $\mathrm{PBE}+U$ electron densities were used to start the calculations with the hybrid functional YS-PBEh, and it was found that for two selected lattice constants, FM1 is more stable than FM2 for all values of $\alpha_{\mathrm{x}}$ that we considered. Since calculations with the YS-PBEh functional are expensive, only the FM1 (and NM) solutions will be considered for YS-PBEh in the following.

The occupation matrix of the FM solution stabilized with LDA and PBE (called FM in the following) does not differ too much from the NM occupation matrix, but shows a more pronounced $f_{x y z}$ character.

In Ref. 12, magnetic moments of about 1 and $0.2 \mu_{\mathrm{B}}$ for the large-volume and small-volume phases, respectively, were obtained with the PBE0 functional. However, we have not been able to stabilize such a solution with a small magnetic moment of $0.2 \mu_{\mathrm{B}}$ (all solutions mentioned above for the FM phase correspond to a spin 
magnetic moment of 1.1-1.4 $\mu_{\mathrm{B}}$ ), neither with $\mathrm{PBE}+U$ nor with YS-PBEh. In particular, the use of the fixedspin moment method ${ }^{62}$ to stabilize such a state has been unsuccessful.

\section{B. Lattice constant and relative energy}

The results for the lattice constants and relative energies are shown in Fig. 2 and Table @ As already shown previously,, 13 LDA strongly underestimates the lattice constant by $\sim 0.3 \AA$ compared with the experimental value of $4.85 \AA$ for the $\alpha$ phase, $\frac{1}{-}$ while the use of a GGA functional such as PBE leads to better agreement, albeit there is still an underestimation of $0.1 \AA$. With LDA and PBE it was also possible to stabilize a FM solution, but only for values of the lattice constant $a$ larger than $\sim 5.1$ $\AA$ as shown in Fig. 2 At the largest value of the lattice constant that we considered $(\sim 5.55 \AA)$, the spin magnetic moment in the unit cell is quite large $\left(\sim 1.4 \mu_{\mathrm{B}}\right.$ for LDA and $\sim 1.6 \mu_{\mathrm{B}}$ for $\left.\mathrm{PBE}\right)$, but then decreases when $a$ gets smaller to finally disappear when the FM curve (smoothly) joins the NM curve at about $5.1 \AA$.

For the LDA/PBE $+U$ functional, several values of $U$ in the range $0-8 \mathrm{eV}$ were considered, and for two of them the results are shown in Fig. 2 and Table I. A value of $U=1.5 \mathrm{eV}$ leads to quite satisfactory results within the PBE $+U$ method. The minima of the NM and FM1 (more stable than FM2) curves are at 4.76 and 5.13 $\AA$, respectively, the latter value being in good agreement with the experimental value of $5.16 \AA^{1}$ for the $\gamma$ phase. More importantly, the NM phase is more stable than the FM1 phase by $\Delta E=-22 \mathrm{meV}$, which is in agreement with the range of values (from -20 to $-30 \mathrm{meV}$ ) deduced from experiment. 32,41 The calculated transition pressure amounts to $P_{t}=-0.5 \mathrm{GPa}$, which seems to be smaller than the experimental value which should be around -1 GPa: 1 Actually, Wang et al ${ }^{20}$ already showed that at $T=0 \mathrm{~K}$, a value of $U=1.6 \mathrm{eV}$ (also with $\mathrm{PBE}+U$ ) leads overall to the most consistent results and in particular to the correct stability ordering. The FM2 and FM3 solutions are less stable than FM1 by 18 and 55 meV, respectively, and the lattice constant for FM3 is shorter than for FM1 and FM2 by $\sim 0.1 \AA$. The results for $\mathrm{LDA}+U$ (Table I) show that the lattice constants of the NM and FM phases are strongly underestimated. We found that for this functional, a value around $U=2.7$ $\mathrm{eV}$ leads to good agreement with experiment for $\Delta E$.

Recently, in Ref. 63 the constrained RPA method was used to calculate the (static) parameters $U$ and $J$ for the early lanthanides. The values for $U-J$ that were obtained for the $\alpha$ and $\gamma$ phases are 3.8 and $4.8 \mathrm{eV}$, respectively (this difference between the two phases is a consequence of their different lattice constants). The results obtained with the average $(4.3 \mathrm{eV})$ of these two values for $U$ in our $\mathrm{PBE}+U$ calculations (we recall that we set $J=0$ ) are given in Fig. [2 and Table I. In contrast to what was obtained with $U=1.5 \mathrm{eV}$, we can see that
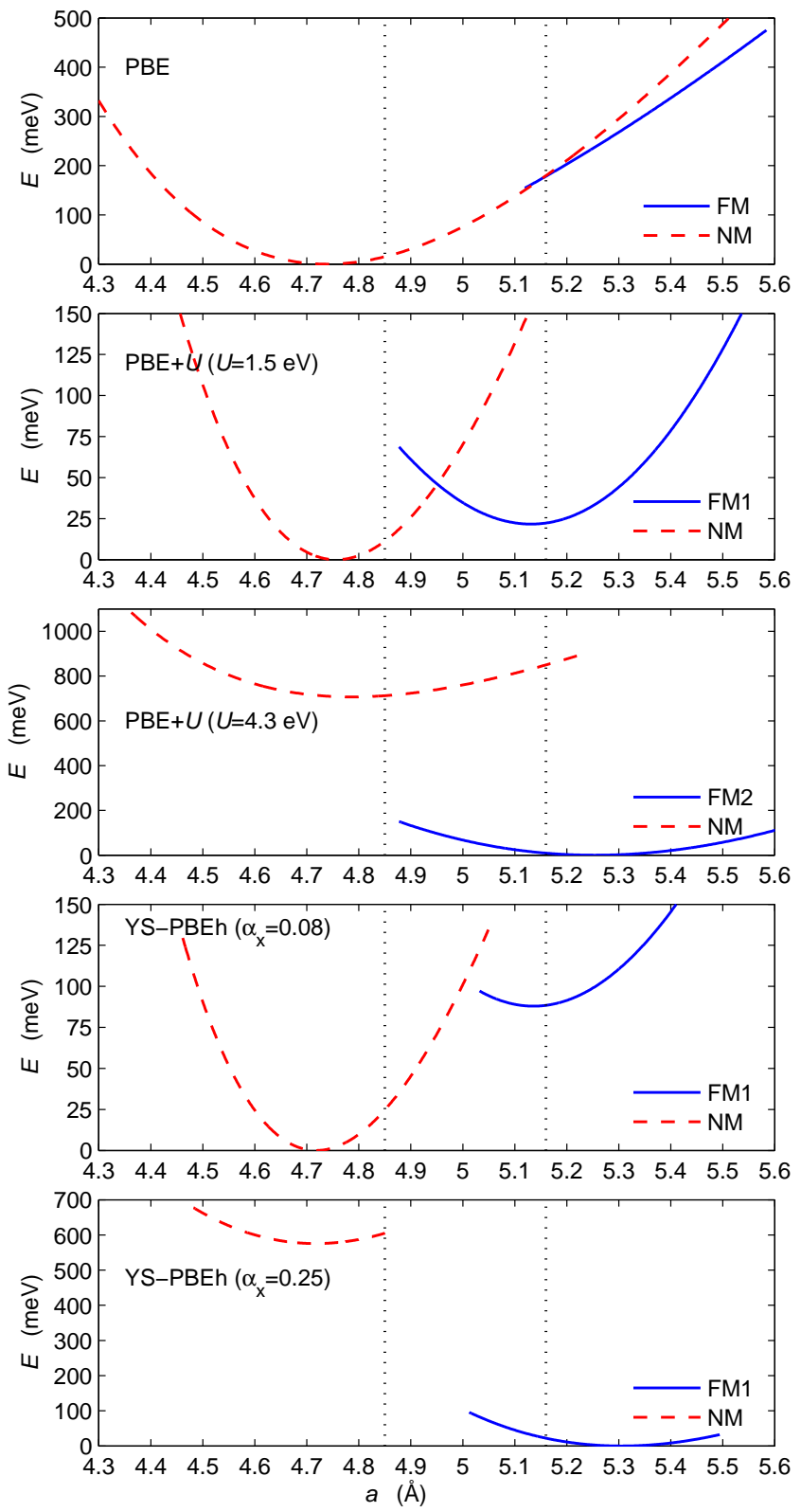

FIG. 2. (Color online) Total energy of the FM (blue solid curve) and NM (red dashed curve) phases of cerium versus the lattice constant calculated with different functionals. The vertical dotted lines indicate the experimental values of the $\alpha$ $(4.85 \AA)$ and $\gamma(5.16 \AA)$ phases. The zero of the energy was set at the minimum of the most stable phase. Note that the scale on the energy axis is different for each functional. The results for $\mathrm{PBE}$ and $\mathrm{PBE}+U$ include $\mathrm{SOC}$ effects.

with $U=4.3 \mathrm{eV}$, the $\mathrm{FM}$ solutions are more stable than the NM one by $600-700 \mathrm{meV}$, which is not the correct stability ordering. Furthermore, the lattice constants of the FM phases are now too large by $0.1 \AA$, while for the $\mathrm{NM}$ phase there is still a sizable underestimation compared to experiment (4.79 $\AA$ with $U=4.3 \mathrm{eV}$ versus $4.85 \AA$ for experiment).

In the $0-0.25$ range of fraction $\alpha_{\mathrm{x}}$ of HF exchange in 
TABLE I. Equilibrium lattice constant $a_{0}$ (in $\AA$ ) and total-energy difference $\Delta E=E^{\mathrm{NM}}-E^{\mathrm{FM}}$ (in meV) between the minima of the FM and NM phases. A negative value of $\Delta E$ indicates that the NM phase is more stable than the FM phase. The results for LDA, PBE, LDA $+U$, and $\mathrm{PBE}+U$ include SOC effects.

\begin{tabular}{|c|c|c|c|c|c|c|c|}
\hline Method & $a_{0}^{\mathrm{NM}}$ & $a_{0}^{\mathrm{FM} 1}$ & $a_{0}^{\mathrm{FM} 2}$ & $a_{0}^{\mathrm{FM} 3}$ & $\Delta E^{\mathrm{FM} 1}$ & $\Delta E^{\mathrm{FM} 2}$ & $\Delta E^{\mathrm{FM} 3}$ \\
\hline LDA & 4.52 & & & & & & \\
\hline PBE & 4.74 & & & & & & \\
\hline $\mathrm{LDA}+U(U=2.7 \mathrm{eV})$ & 4.58 & & & 4.91 & & & -32 \\
\hline $\mathrm{LDA}+U(U=4.3 \mathrm{eV})$ & 4.60 & & & 5.00 & & & 362 \\
\hline $\mathrm{PBE}+U(U=1.5 \mathrm{eV})$ & 4.76 & 5.13 & 5.16 & 5.06 & -22 & -40 & -77 \\
\hline $\mathrm{PBE}+U(U=4.3 \mathrm{eV})$ & 4.79 & 5.26 & 5.25 & 5.25 & 692 & 707 & 616 \\
\hline YS-PBEh $\left(\alpha_{\mathrm{x}}=0.08\right)$ & 4.72 & 5.14 & & & -88 & & \\
\hline YS-PBEh $\left(\alpha_{\mathrm{x}}=0.25\right)$ & 4.72 & 5.31 & & & 576 & & \\
\hline Expt. & $4.85^{\mathrm{a}}$ & & $5.16^{\mathrm{a}}$ & & & -20 to & \\
\hline
\end{tabular}

a Reference 1 .

b References 32 and 41 .

TABLE II. Spin magnetic moment in the unit cell $M_{\text {spin,cell }}$ (in $\mu_{\mathrm{B}}$ ), orbital magnetic moment of the $4 f$-electrons $M_{\mathrm{orb}, 4 f}$ (in $\left.\mu_{\mathrm{B}}\right)$, and number of $4 f$-electrons $n_{4 f}$ inside the atomic sphere $\left(R_{\mathrm{MT}}^{\mathrm{Ce}}=2.2 \mathrm{bohr}\right)$. The results for LDA, PBE, LDA $+U$, and $\mathrm{PBE}+U$ include SOC effects.

\begin{tabular}{|c|c|c|c|c|c|c|c|c|c|c|}
\hline Method & $M_{\mathrm{spin}, \mathrm{cell}}^{\mathrm{FM} 1}$ & $M_{\text {spin,cell }}^{\mathrm{FM}}$ & $M_{\mathrm{spin}, \mathrm{cell}}^{\mathrm{FM} 3}$ & $M_{\mathrm{orb}, 4 f}^{\mathrm{FM} 1}$ & $M_{\mathrm{orb}, 4 f}^{\mathrm{FM} 2}$ & $M_{\mathrm{orb}, 4 f}^{\mathrm{FM} 3}$ & $n_{4 f}^{\mathrm{NM}}$ & $n_{4 f}^{\mathrm{FM} 1}$ & $n_{4 f}^{\mathrm{FM} 2}$ & $n_{4 f}^{\mathrm{FM} 3}$ \\
\hline $\mathrm{LDA}$ & & & & & & & 0.95 & & & \\
\hline PBE & & & & & & & 0.91 & & & \\
\hline $\mathrm{LDA}+U(U=2.7 \mathrm{eV})$ & & & 1.1 & & & -1.6 & 0.78 & & & 0.95 \\
\hline $\mathrm{LDA}+U(U=4.3 \mathrm{eV})$ & & & 1.2 & & & -1.6 & 0.69 & & & 0.97 \\
\hline $\mathrm{PBE}+U(U=1.5 \mathrm{eV})$ & 1.2 & 1.4 & 1.2 & -0.5 & -0.6 & -1.5 & 0.82 & 0.95 & 0.99 & 0.93 \\
\hline $\mathrm{PBE}+U(U=4.3 \mathrm{eV})$ & 1.2 & 1.3 & 1.2 & -0.5 & -0.5 & -1.8 & 0.65 & 0.96 & 0.97 & 0.96 \\
\hline YS-PBEh $\left(\alpha_{\mathrm{x}}=0.08\right)$ & 1.2 & & & & & & 0.81 & 0.93 & & \\
\hline YS-PBEh $\left(\alpha_{\mathrm{x}}=0.25\right)$ & 1.1 & & & & & & 0.61 & 0.94 & & \\
\hline
\end{tabular}

YS-PBEh, two values are of interest and the corresponding results are shown in Fig. 2 and Table [ $\alpha_{\mathrm{x}}=0.08$ is a value which leads to rather similar results as $\mathrm{PBE}+U$ with $U=1.5 \mathrm{eV}$ and therefore in fair agreement with experiment, except for the lattice constant of the $\alpha$ phase which is still underestimated by about $0.1 \AA$. The energy difference between the NM and FM1 phases is $\Delta E=-88$ meV, which seems to be slightly too large in magnitude but still reasonable at a qualitative level, while the transition pressure is at $P_{t}=-1.7 \mathrm{GPa}$. The value $\alpha_{\mathrm{x}}=0.25$ is a non-empirical value which was deduced from perturbation theory arguments (see Ref. 64). The results obtained with $\alpha_{\mathrm{x}}=0.25$ show a rather large overestimation of $0.15 \AA$ for the lattice constant of the FM1 phase and the wrong stability ordering $(\Delta E=576 \mathrm{meV})$ of the two phases (similar to $\mathrm{PBE}+U$ with $U=4.3 \mathrm{eV}$ ). Note that, these results with $\alpha_{\mathrm{x}}=0.25$ agree with the HSE06 results of Casadei et $a l, 12$ but with the difference that in their $\alpha$ phase a non-zero magnetic moment of about 0.2 $\mu_{\mathrm{B}}$ is obtained. It is also worth mentioning that their RPA results show the right stability ordering, however the lattice constants are too small, in particular for the $\alpha$ phase (underestimations of 0.4 and $0.1 \AA$ for the $\alpha$ and $\gamma$ phases, respectively).

In Ref. 24, the results of calculations obtained with the $\mathrm{LDA}+\mathrm{GA}$ and LDA+DMFT methods at the temperature $T=0 \mathrm{~K}$ were reported. It was concluded that the phase transition (indicated by a change of sign in the bulk modulus) can be observed only if SOC is included in the calculations. As already mentioned in Sec. II SOC has very little influence on the results of our calculations.

We finish this section by mentioning that the FM2 and FM3 solutions (only reachable if cubic symmetry is broken) lead to structural distortion (not included in the results shown in Table I and Fig. 2). By considering tetragonal distortion along the $z$-direction (i.e., $c$-axis), we calculated a ratio $c / a$ of about 0.97 and a lowering of the total energy (with respect to the cubic unit cell) of the order of $2 \mathrm{meV}$, which is one order of magnitude smaller than the relative stability of the $\alpha$ and $\gamma$ phases. The NM and FM1 solutions (including SOC) lead to no distortion. However, we mention that the observed distortions for the FM2 and FM3 solutions are artifacts in the sense that Ce is paramagnetic (and not ferromagnetic as in our work) such that the randomness of the orientations of the local moments would cancel any structural 


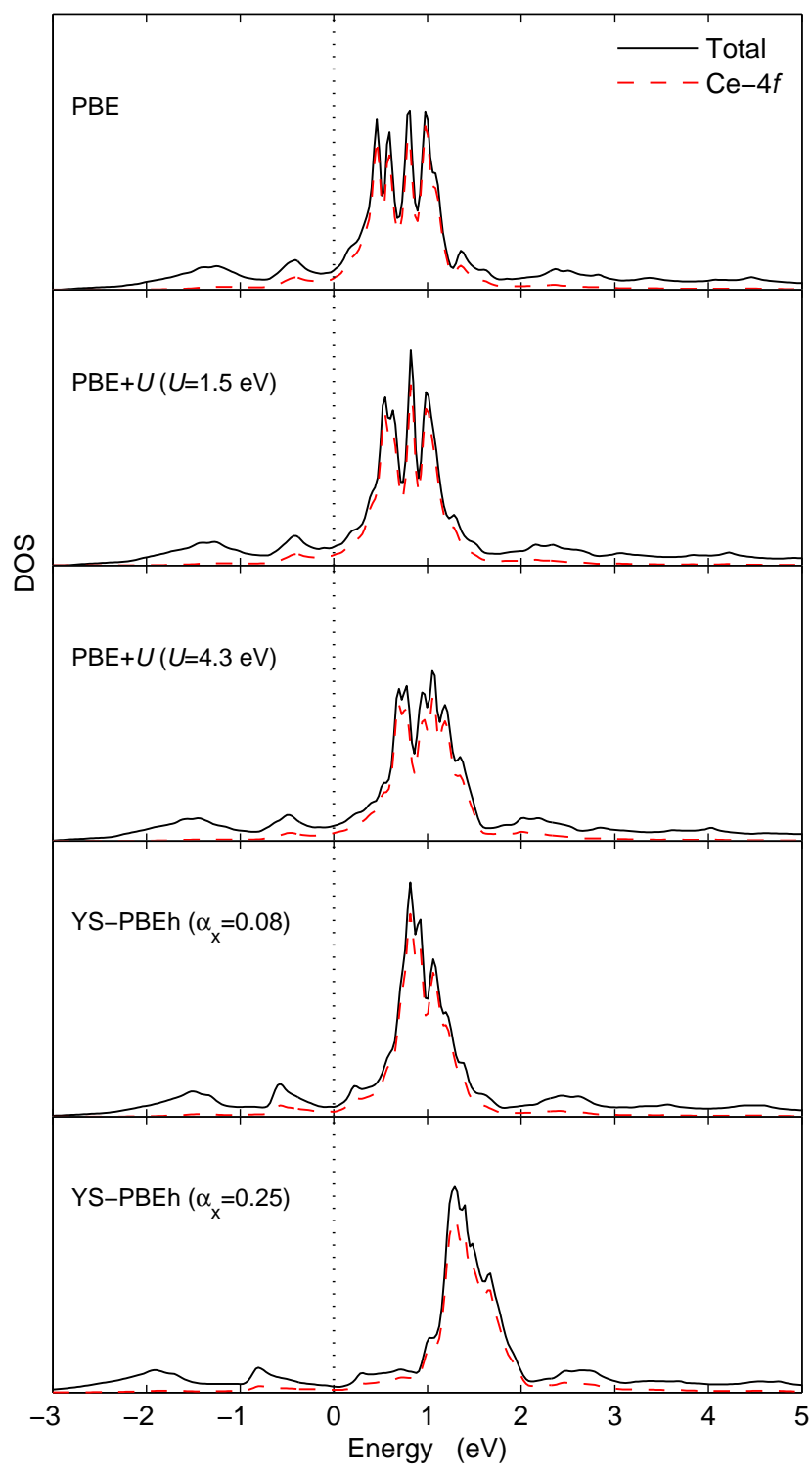

FIG. 3. (Color online) Total and $4 f$ one-spin density of states for the NM phase of cerium. The results for $\mathrm{PBE}$ and $\mathrm{PBE}+U$ include SOC effects. The Fermi energy is set at $E=0 \mathrm{eV}$.

distortion.

\section{Electronic structure}

Photoemission spectroscopy (PES $)^{\underline{4} \underline{\underline{6}}}$ and inverse PES 4 experiments have shown that lower and upper Hubbard bands are present in the $\alpha$ and $\gamma$ phases, which indicates that the $4 f$-electrons are strongly correlated in both phases. The lower Hubbard band is situated at about -2.2 and $-2 \mathrm{eV}$ below the Fermi energy in the $\alpha$ and $\gamma$ phases, respectively, while the upper Hubbard band is at $4 \mathrm{eV}$ above the Fermi energy in both phases. In addition, in the $\alpha$ phase a quasiparticle peak (Kondo

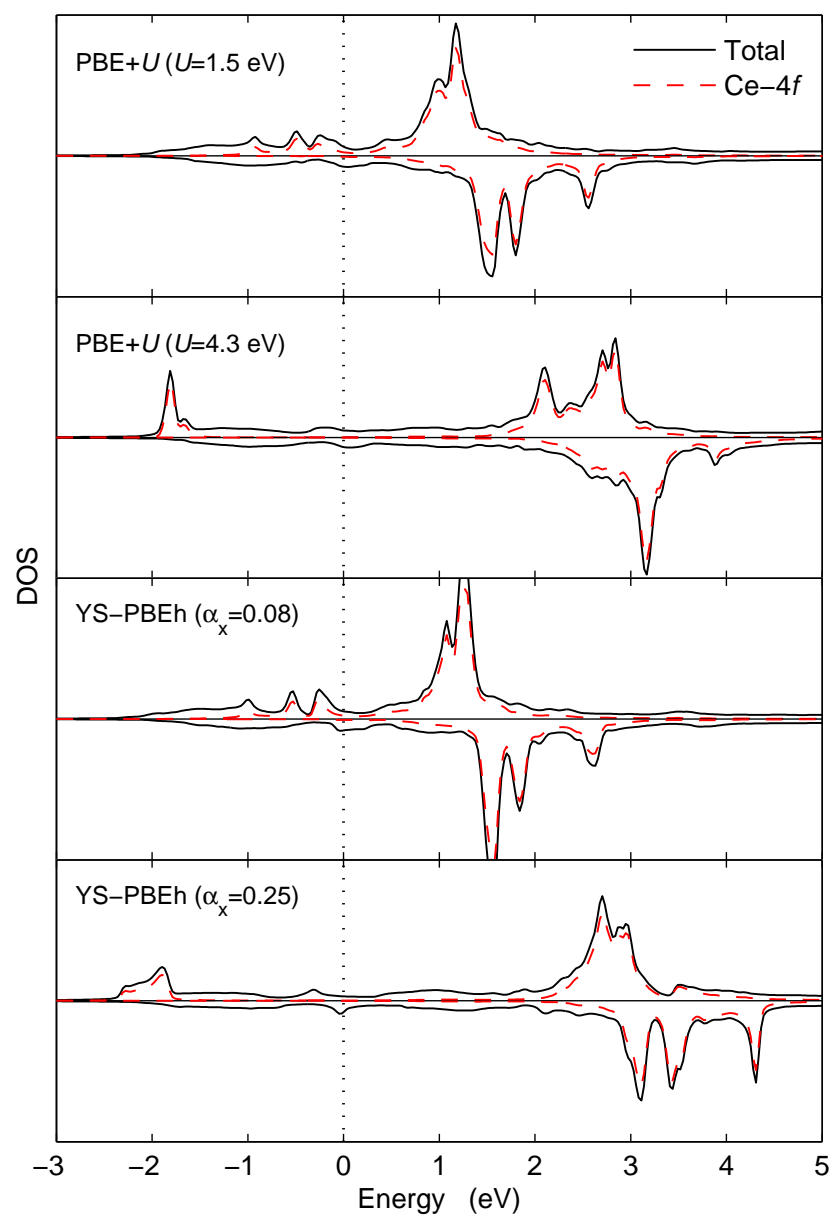

FIG. 4. (Color online) Total and $4 f$ spin-majority (upwards) and spin-minority (downwards) density of states for the FM phase of cerium. The FM state is FM2 for $\mathrm{PBE}+U$ with $U=4.3 \mathrm{eV}$ and FM1 in the other cases. The results for $\mathrm{PBE}+U$ include SOC effects. The Fermi energy is set at $E=0 \mathrm{eV}$.

resonance) is observed at the Fermi energy.

Figures 3 and 4 show the calculated DOS obtained with the PBE-based methods of the NM and FM phases, respectively. In the NM case, we can see that the occupied part of the Ce- $4 f$ partial DOS is rather flat and extends from -1 to $0 \mathrm{eV}$ below the Fermi energy, which is in disagreement with experiment, and actually, there is no clearly separated lower and upper Hubbard bands. In general, the features of the NM DOS are pretty similar among all considered functionals since the value of $U$ or $\alpha_{\mathrm{x}}$ seems to have a moderate influence on the position of the Ce- $4 f$ partial DOS. Note that since in the NM phase, the main part of the $4 f$ DOS is situated just above the Fermi energy, it is tempting to assign it to the observed quasiparticle peak, however, it is questionable wether such a feature which originates from many-body effects can be described by one-electron methods.

Figure 4 (FM DOS) shows that for the small value of $U(1.5 \mathrm{eV})$ or $\alpha_{\mathrm{x}}(0.08)$, the occupied Ce- $4 f$ DOS (the 


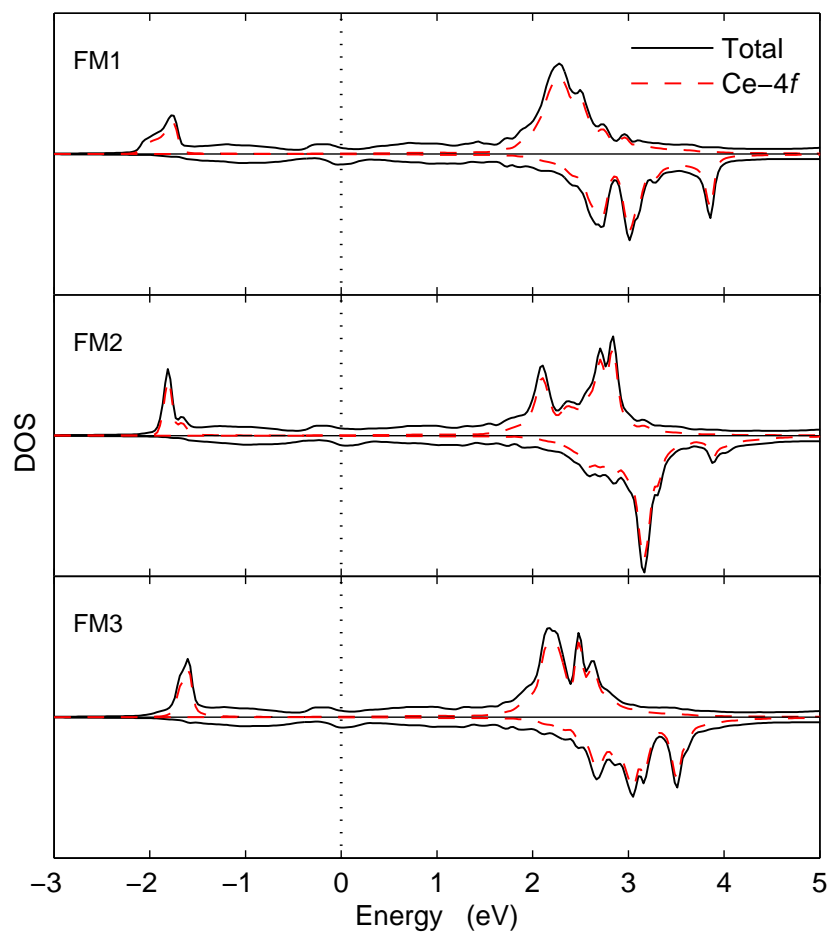

FIG. 5. (Color online) Total and $4 f$ spin-majority (upwards) and spin-minority (downwards) density of states for the three FM phases of cerium as obtained from $\mathrm{PBE}+U(U=4.3 \mathrm{eV})$ with SOC. The Fermi energy is set at $E=0 \mathrm{eV}$.

lower Hubbard band) is relatively flat and in the range $[-1,0] \mathrm{eV}$ (as for the NM phase), while for larger $U(4.3$ $\mathrm{eV})$ or $\alpha_{\mathrm{x}}(0.25)$, the Ce- $4 f$ DOS is sharper and is shifted down at $-2 \mathrm{eV}$ below the Fermi energy, which is in good agreement with experiment. As previously shown in Refs. 18 and 19 , $\mathrm{LDA}+U$ with $U-J=5.4 \mathrm{eV}$ puts the lower Hubbard band at $-2.5 \mathrm{eV}$ in the $\gamma$ phase. We can see that larger values of $U$ or $\alpha_{\mathrm{x}}$ also lead to a more correct position of the upper Hubbard band.

With $\mathrm{PBE}+U$, the small sensitivity of the Ce- $4 f$ DOS to $U$ in the NM case can be simply explained by the fact that the diagonal terms of the occupation matrix [see Eq. (A4)] are more or less of equal magnitude, such that the shift due to the orbital-dependent $U$-potential [see Eq. (25) in Ref. 65] is similar wether the $4 f$ orbital is below or above the Fermi energy. In the FM case, the occupied $4 f$-orbital corresponds to one (or two) particular value of $m$ [Eqs. (A1)-(A3)], which allows the $U$-potential to shift [by $\sim(U-J) / 2$ ] the lower and upper Hubbard bands in opposite directions. With the hybrid functional YS-PBEh, whose potential is also orbitaldependent, a similar mechanism occurs. As already observed for the lattice constants and relative energies, the $\mathrm{PBE}+U$ and YS-PBEh results are pretty similar for the electronic structure, too.

In order to show the effect of orbital occupation on the DOS, we show in Fig. 5 the DOS of the three FM states, all obtained from the same method $(\mathrm{PBE}+U$ with $U=4.3 \mathrm{eV}$ and including SOC). As we can see, the symmetry of the occupied $4 f$ orbital has overall little influence on the position of the center of mass of the lower and upper Hubbard bands.

Overall, it seems that it is not possible to reproduce all important features seen in the (inverse) PES experiments. More specifically, none of the one-electron methods that we have considered is able to yield a spectrum for the $\alpha$ phase showing (simultaneously) the Hubbard bands and the quasiparticle peak. In the recent study of Sakuma et al. (Ref. 35), it has been shown that the non-self consistent $G W$ method ( $G W$ on top of LDA) can also not reproduce correctly all main features of the experimental spectrum (no lower Hubbard band at $-2 \mathrm{eV}$ and presence of a quasiparticle peak also in the $\gamma$ phase). By now, only LDA+DMFT, which properly takes into account many-body effects, is able to yield good agreement with experiment, $25,26,28-30,32$ It is worth to mention that our calculated DOS in the NM phase looks rather similar to the LDA+DMFT spectrum obtained at very small lattice constants (see Refs. 26 and 29).

From neutron inelastic-scattering experiment, $\frac{66}{6}$ it was inferred that the number of $4 f$-electrons $n_{4 f}$ is smaller in the $\alpha$ phase than in the $\gamma$ phase by $0.2 \pm 0.1$. The values of $n_{4 f}$ inside the atomic sphere $\left(R_{\mathrm{MT}}^{\mathrm{Ce}}=2.2 \mathrm{bohr}\right)$ shown in Table Ireproduce this trend, albeit the difference $n_{4 f}^{\mathrm{FM}}-$ $n_{4 f}^{\mathrm{NM}}$ seems too be at the limit of being too large (0.33) for PBE $+U$ with $U=4.3 \mathrm{eV}$ and YS-PBEh with $\alpha_{\mathrm{x}}=0.25$. On the side of LDA+DMFT, some discrepancies among the various studies were obtained. For instance, while in Ref. 32, $n_{4 f}$ was calculated to be larger in the $\alpha$ phase for temperatures ranging from 400 to $1600 \mathrm{~K}$ (a monotonous increase upon compression is obtained), the opposite trend (and with a non-monotonous behavior of $n_{4 f}$ ) was obtained in Ref. 27 for temperatures below roughly $1000 \mathrm{~K}$. Note, however, that the value of $n_{4 f}$, and possibly the trend in the variation due to volume change, depends on the basis set and more particularly on the size of the atomic sphere.

The results for the magnetic moments are shown in Table [II. We can see that for the spin moment in the unit cell $M_{\text {spin,cell }}, \mathrm{PBE}+U$ and YS-PBEh lead to a value of $1.1-1.2 \mu_{\mathrm{B}}$ for the FM1 solution, whatever is the value of $U$ or $\alpha_{\mathrm{x}}$. The values are similar for FM3, while they are slightly larger by $0.1-0.2 \mu_{\mathrm{B}}$ for FM2. The contribution to $M_{\text {spin,cell }}$ coming from inside the atomic sphere amounts to $0.9-1.0 \mu_{\mathrm{B}}$ and the rest comes from the interstitial region. SOC induces an orbital moment and for the $4 f$ shell $\left(M_{\text {orb }, 4 f}\right)$, we can see that for FM1 and FM2 the values are similar $\left(-0.5 \mu_{\mathrm{B}}\right)$, while they are much larger for FM3 since this solution corresponds mainly to an electron in the $Y_{3}^{-2}$ orbital. 


\section{SUMMARY}

The purpose of this work has been to study with KS and mixed KS/HF methods the FM and NM phases of elemental cerium. Several types of functionals were considered and for two of them, DFT $+U$ and YS-PBEh, it was possible to get a minimum in the total-energy curve for both phases without imposing any constraint on the spin symmetry. The parameters $U$ and $\alpha_{\mathrm{x}}$ in these functionals were varied in order to examine their influence on the properties.

In order to compare our results with experiment, we have supposed that our NM and FM solutions correspond to the $\alpha$ and $\gamma$ phases that were observed experimentally. We have shown that the correct stability ordering of the $\alpha$ and $\gamma$ phases can be obtained only for small values of $U$ or $\alpha_{\mathrm{x}}$. On the other hand, the electronic structure is better reproduced with larger values of $U$ or $\alpha_{\mathrm{x}}$, but none of the considered methods is able to give an overall correct description of the electronic structure. In particular, up to now only the many-body LDA+DMFT method has been able to reproduce the Hubbard bands and quasiparticle peak in the $\alpha$ phase. In this respect, it would be very interesting to know how would perform the HF+RPA method when applied self-consistently as a one-electron method 67

\section{ACKNOWLEDGMENTS}

This work was supported by the project SFB-F41 (ViCoM) of the Austrian Science Fund.

\section{Appendix A: Occupation Matrix}

In this appendix, the majority-spin $4 f$ occupation matrices $n_{m m^{\prime}}$ of the FM and NM solutions (at their respective equilibrium volume) obtained from $\mathrm{PBE}+U$ with $U=4.3 \mathrm{eV}$ and including SOC are given.

1 D. C. Koskenmaki and K. A. Gschneidner, Jr., in Handbook on the Physics and Chemistry of Rare Earths, edited by K. A. Gschneidner, Jr. and L. Eyring (North-Holland, Amsterdam, 1978), Vol. 1, p. 337.

2 B. Johansson, Philos. Mag. 30, 469 (1974).

3 J. W. Allen and R. M. Martin, Phys. Rev. Lett. 49, 1106 (1982).

4 E. Wuilloud, H. R. Moser, W.-D. Schneider, and Y. Baer, Phys. Rev. B 28, R7354 (1983).

5 D. M. Wieliczka, C. G. Olson, and D. W. Lynch, Phys. Rev. B 29, 3028 (1984).

6 E. Weschke, C. Laubschat, T. Simmons, M. Domke, O. Strebel, and G. Kaindl, Phys. Rev. B 44, 8304 (1991).

7 W. E. Pickett, A. J. Freeman, and D. D. Koelling, Phys. Rev. B 23, 1266 (1981).
FM1:

$$
\left(\begin{array}{ccccccc}
0.00 & 0.00 & 0.00 & 0.00 & 0.00 & 0.00 & 0.00 \\
0.00 & 0.59 & 0.00 & 0.00 & 0.00 & -0.44 & 0.00 \\
0.00 & 0.00 & 0.00 & 0.00 & 0.00 & 0.00 & 0.00 \\
0.00 & 0.00 & 0.00 & 0.01 & 0.00 & 0.00 & 0.00 \\
0.00 & 0.00 & 0.00 & 0.00 & 0.00 & 0.00 & 0.00 \\
0.00 & -0.44 & 0.00 & 0.00 & 0.00 & 0.33 & 0.00 \\
0.00 & 0.00 & 0.00 & 0.00 & 0.00 & 0.00 & 0.00
\end{array}\right)
$$

FM2:

$$
\left(\begin{array}{lllllll}
0.00 & 0.00 & 0.00 & 0.00 & 0.00 & 0.00 & 0.00 \\
0.00 & 0.59 & 0.00 & 0.00 & 0.00 & 0.44 & 0.00 \\
0.00 & 0.00 & 0.00 & 0.00 & 0.00 & 0.00 & 0.00 \\
0.00 & 0.00 & 0.00 & 0.00 & 0.00 & 0.00 & 0.00 \\
0.00 & 0.00 & 0.00 & 0.00 & 0.00 & 0.00 & 0.00 \\
0.00 & 0.44 & 0.00 & 0.00 & 0.00 & 0.34 & 0.00 \\
0.00 & 0.00 & 0.00 & 0.00 & 0.00 & 0.00 & 0.00
\end{array}\right)
$$

FM3:

$$
\left(\begin{array}{ccccccc}
0.00 & 0.00 & 0.00 & 0.00 & 0.00 & 0.00 & 0.00 \\
0.00 & 0.92 & 0.00 & 0.00 & 0.00 & -0.02 & 0.00 \\
0.00 & 0.00 & 0.00 & 0.00 & 0.00 & 0.00 & 0.00 \\
0.00 & 0.00 & 0.00 & 0.01 & 0.00 & 0.00 & 0.00 \\
0.00 & 0.00 & 0.00 & 0.00 & 0.00 & 0.00 & 0.00 \\
0.00 & -0.02 & 0.00 & 0.00 & 0.00 & 0.00 & 0.00 \\
0.00 & 0.00 & 0.01 & 0.00 & 0.00 & 0.00 & 0.00
\end{array}\right)
$$

NM:

$$
\left(\begin{array}{ccccccc}
0.05 & 0.00 & 0.00 & 0.00 & 0.03 & 0.00 & 0.00 \\
0.00 & 0.08 & 0.00 & 0.00 & 0.00 & -0.06 & 0.00 \\
0.00 & 0.00 & 0.03 & 0.00 & 0.00 & 0.00 & 0.02 \\
0.00 & 0.00 & 0.00 & 0.06 & 0.00 & 0.00 & 0.00 \\
0.03 & 0.00 & 0.00 & 0.00 & 0.03 & 0.00 & 0.00 \\
0.00 & -0.06 & 0.00 & 0.00 & 0.00 & 0.06 & 0.00 \\
0.00 & 0.00 & 0.02 & 0.00 & 0.00 & 0.00 & 0.03
\end{array}\right)
$$

8 B. Johansson, I. A. Abrikosov, M. Aldén, A. V. Ruban, and H. L. Skriver, Phys. Rev. Lett. 74, 2335 (1995).

${ }^{9}$ P. Söderlind, O. Eriksson, J. Trygg, B. Johansson, and J. M. Wills, Phys. Rev. B 51, 4618 (1995).

10 T. Jarlborg, E. G. Moroni, and G. Grimvall, Phys. Rev. B 55, 1288 (1997).

11 T. Jarlborg, Phys. Rev. B 58, 9599 (1998).

12 M. Casadei, X. Ren, P. Rinke, A. Rubio, and M. Scheffler, Phys. Rev. Lett. 109, 146402 (2012).

13 Z. Szotek, W. M. Temmerman, and H. Winter, Phys. Rev. Lett. 72, 1244 (1994).

14 A. Svane, Phys. Rev. Lett. 72, 1248 (1994).

15 A. Svane, Phys. Rev. B 53, 4275 (1996).

16 J. Lægsgaard and A. Svane, Phys. Rev. B 59, 3450 (1999).

17 M. Lüders, A. Ernst, M. Däne, Z. Szotek, A. Svane, D. 
Ködderitzsch, W. Hergert, B. L. Györffy, and W. M. Temmerman, Phys. Rev. B 71, 205109 (2005).

18 A. B. Shick, W. E. Pickett, A. I. Liechtenstein, J. Electron Spectrosc. Relat. Phenom. 114-116, 753 (2001).

19 B. Amadon, F. Jollet, and M. Torrent, Phys. Rev. B 77, 155104 (2008).

${ }^{20}$ Y. Wang, L. G. Hector, Jr., H. Zhang, S. L. Shang, L. Q. Chen, and Z. K. Liu, Phys. Rev. B 78, 104113 (2008).

21 B. Amadon, J. Phys.: Condens. Matter 24, 075604 (2012).

22 O. Eriksson, M. S. S. Brooks, and B. Johansson, Phys. Rev. B 41, 7311 (1990).

23 M.-F. Tian, X. Deng, Z. Fang, X. Dai, Phys. Rev. B 84, 205124 (2011).

24 N. Lanatà, Y.-X. Yao, C.-Z. Wang, K.-M. Ho, J. Schmalian, K. Haule, and G. Kotliar, Phys. Rev. Lett. 111, 196801 (2013).

25 M. B. Zölfl, I. A. Nekrasov, T. Pruschke, V. I. Anisimov, and J. Keller, Phys. Rev. Lett. 87, 276403 (2001).

${ }^{26}$ K. Held, A. K. McMahan, R. T. Scalettar, Phys. Rev. Lett. 87, 276404 (2001).

27 A. K. McMahan, K. Held, and R. T. Scalettar, Phys. Rev. B 67, 075108 (2003).

${ }^{28}$ K. Haule, V. Oudovenko, S. Y. Savrasov, and G. Kotliar Phys. Rev. Lett. 94, 036401 (2005).

29 A. K. McMahan, Phys. Rev. B 72, 115125 (2005).

30 O. Sakai, Y. Shimizu, and Y. Kaneta, J. Phys. Soc. Jpn. 74, 2517 (2005).

31 J.-P. Rueff, J.-P. Itié, M. Taguchi, C. F. Hague, J.-M. Mariot, R. Delaunay, J.-P. Kappler, and N. Jaouen, Phys. Rev. Lett. 96, 237403 (2006).

32 B. Amadon, S. Biermann, A. Georges, and F. Aryasetiawan, Phys. Rev. Lett. 96, 066402 (2006).

${ }^{33}$ L. V. Pourovskii, B. Amadon, S. Biermann, and A. Georges, Phys. Rev. B 76, 235101 (2007).

34 B. Chakrabarti, M. E. Pezzoli, G. Sordi, K. Haule, and G. Kotliar, Phys. Rev. B 89, 125113 (2014).

35 R. Sakuma, T. Miyake, and F. Aryasetiawan, Phys. Rev. B 86, 245126 (2012).

36 A. V. Nikolaev and A. V. Tsvyashchenko, Phys.-Usp. 55, 657 (2012).

37 W. Kohn and L. J. Sham, Phys. Rev. 140, A1133 (1965).

38 P. Hohenberg and W. Kohn, Phys. Rev. 136, B864 (1964).

${ }^{39}$ K. Terakura, T. Oguchi, A. R. Williams, and J. Kübler, Phys. Rev. B 30, 4734 (1984).

40 M. J. Lipp, A. P. Sorini, J. Bradley, B. Maddox, K. T. Moore, H. Cynn, T. P. Devereaux, Y. Xiao, P. Chow, W. J. Evans, Phys. Rev. Lett. 109, 195705 (2012).

41 F. Decremps, L. Belhadi, D. L. Farber, K. T. Moore, F. Occelli, M. Gauthier, A. Polian, D. Antonangeli, C. M. Aracne-Ruddle, and B. Amadon, Phys. Rev. Lett. 106, 065701 (2011).

42 J. Heyd, G. E. Scuseria, and M. Ernzerhof, J. Chem. Phys. 118, 8207 (2003); 124, 219906 (2006).
43 A. V. Krukau, O. A. Vydrov, A. F. Izmaylov, and G. E. Scuseria, J. Chem. Phys. 125, 224106 (2006).

44 M. Ernzerhof and G. E. Scuseria, J. Chem. Phys. 110, 5029 (1999).

45 C. Adamo and V. Barone, J. Chem. Phys. 110, 6158 (1999).

46 P. Blaha, K. Schwarz, G. K. H. Madsen, D. Kvasnicka, and J. Luitz, WIEn2K: An Augmented Plane Wave plus Local Orbitals Program for Calculating Crystal Properties, edited by K. Schwarz (Vienna University of Technology, Austria, 2001).

47 D. J. Singh and L. Nordström, Planewaves, Pseudopotentials and the $L A P W$ Method, 2nd ed. (Springer, Berlin, 2006).

48 F. Tran and P. Blaha, Phys. Rev. B 83, 235118 (2011).

49 J. P. Perdew and Y. Wang, Phys. Rev. B 45, 13244 (1992).

50 J. P. Perdew, K. Burke, and M. Ernzerhof, Phys. Rev. Lett. 77, 3865 (1996); 78, 1396 (1997).

51 A. I. Liechtenstein, V. I. Anisimov, and J. Zaanen, Phys. Rev. B 52, R5467 (1995).

52 A. B. Shick, A. I. Liechtenstein, and W. E. Pickett, Phys. Rev. B 60, 10763 (1999).

53 V. I. Anisimov, I. V. Solovyev, M. A. Korotin, M. T. Czyżyk, and G. A. Sawatzky, Phys. Rev. B 48, 16929 (1993).

54 M. T. Czyżyk and G. A. Sawatzky, Phys. Rev. B 49, 14211 (1994).

55 D. M. Bylander and L. Kleinman, Phys. Rev. B 41, 7868 (1990).

56 A. S. Botana, F. Tran, V. Pardo, D. Baldomir, and P. Blaha, Phys. Rev. B 85, 235118 (2012).

57 F. Tran, D. Koller, and P. Blaha, Phys. Rev. B 86, 134406 (2012).

58 R. Laskowski, P. Blaha, and F. Tran, Phys. Rev. B 87, 195130 (2013).

59 D. Koller, P. Blaha, and F. Tran, J. Phys.: Condens. Matter 25, 435503 (2013).

${ }^{60}$ L. O. Wagner, E. M. Stoudenmire, K. Burke, and S. R. White, Phys. Rev. Lett. 111, 093003 (2013); 112, 019901(E) (2014).

61 A. Schrön and F. Bechstedt, J. Phys.: Condens. Matter 25, 486002 (2013).

62 K. Schwarz and P. Mohn, J. Phys. F: Met. Phys. 14, L129 (1984).

63 F. Nilsson, R. Sakuma, and F. Aryasetiawan, Phys. Rev. B 88, 125123 (2013).

64 J. P. Perdew, M. Ernzerhof, and K. Burke, J. Chem. Phys. 105, 9982 (1996).

65 E. R. Ylvisaker, W. E. Pickett, and K. Koepernik, Phys. Rev. B 79, 035103 (2009).

66 A. P. Murani, Z. A. Bowden, A. D. Taylor, R. Osborn, W. G. Marshall, Phys. Rev. B 48, 13981 (1993).

67 T. Kotani and H. Akai, J. Magn. Magn. Mater. 177-181, 569 (1998). 